\title{
Funções executivas na dependência de crack: um estudo de caso
}

Funciones ejecutivas y dependencia del crack: un estudio de caso

Fonctions exécutives et dépendance à la cocaïnelau crack: une étude de cas

Executive functions on the crack dependence: a case study

\section{Hosana Alves Gonçalves ${ }^{1}$, Caroline de Oliveira Cardoso ${ }^{1}, \&$ Renata Brasil Araújo ${ }^{2}$}

${ }^{1}$ Pontifícia Universidade Católica do Rio Grande do Sul, Brasil. ${ }^{2}$ Hospital Psiquiátrico São Pedro, Brasil.

\section{Resumo}

\begin{abstract}
A intersecção entre neuropsicologia e psicopatologia ainda é incipiente, principalmente quando se trata do entendimento específico de quais subcomponentes das funções executivas (FE) podem ser afetados por um quadro neuropsiquiátrico como a dependência química. O presente estudo teve como objetivo caracterizar o perfil cognitivo de processamento executivo em um dependente de crack. Uma bateria flexível de testes que avaliam as FE foi utilizada: o teste Wisconsin de Classificação de Cartas - 48 cartões, o Iowa Gambling Task, o Hayling Test e as três modalidades de fluência verbal da Bateria Montreal de Avaliação da Comunicação. Os resultados sugerem uma preservação da maioria dos componentes executivos, como inibição e iniciação verbal, monitoramento, planejamento e flexibilidade cognitiva, porém, parece haver um déficit no componente tomada de decisão. O tempo de abstinência pode ter contribuído para o bom desempenho do paciente na maioria das tarefas. Estudos de grupo ou de casos múltiplos devem ser conduzidos para melhor compreensão da relação de fatores da dependência química com o desempenho em tarefas de FE.

Palavras-chave: Avaliação neuropsicológica; funções executivas; dependência de substâncias.
\end{abstract}

\section{Resumen}

La interrelación entre neuropsicología y psicopatología es todavía incipiente, fundamentalmente cuando se trata de la comprensión de los componentes de las funciones ejecutivas (FE) que pueden estar afectados por un cuadro neuropsiquiátrico como la dependencia química. El presente estudio tiene por objetivo caracterizar el perfil cognitivo de procesamiento ejecutivo en un dependiente al crack. Se utilizó una batería flexible de tests que evalúan las FE. La misma consta del test de Wisconsin de 48 cartas, del Iowa Gambling Task, del Test de Hayling y de las tres modalidades de fluencia verbal de la Batería Montréal de Evaluación de la Comunicación. Los resultados sugieren la preservación de la mayoría de los componentes ejecutivos, como la inhibición y la iniciación verbales, el monitoreo, la planificación y la flexibilidad cognitiva. Sin embargo, el paciente parece presentar un déficit en el componente de la toma de decisiones. El tiempo de abstinencia puede haber contribuido al buen desempeño del paciente en la mayoría de las tareas. Deberían realizarse estudios de grupo o de casos múltiples para comprender más acabadamente la relación entre los factores de la dependencia química y el desempeño en tareas de FE.

Palavras-clave: Evaluación neuropsicológica; funciones ejecutivas; dependencia de sustancias.

\section{Résumé}

La zone de chevauchement entre la neuropsychologie et la psychopathologie est encore peu décrite, en particulier lorsqu'il s'agit de comprendre les composantes spécifiques aux fonctions exécutives (FE) affectées par une maladie neuropsychiatrique telle l'addiction aux drogues. La présente étude vise à caractériser le profil de traitement exécutif dans un cas d'addiction à la cocaïne/crack. Une batterie flexible des FE a été utilisée : Wisconsin Card Sorting Test - 48 cartes, la tâche de jeu de l'Iowa, le Hayling Test ainsi que les trois modalités de fluence verbale de la Batterie Montréal d'Évaluation de la Communication. Les résultats suggèrent une préservation de la majorité des composants des FE, comme l'initiation et l'inhibition verbale, l'attention, la planification et la flexibilité cognitive, alors qu'un déficit semble présent dans la prise de décision. La durée d'abstinence du patient pourrait avoir contribué à des performances préservées dans la plupart des tâches. Afin de mieux comprendre les dissociations des processus exécutifs dans les troubles neuropsychiatriques d'addiction à la drogue, davantage d'études de cas multiples et d'études de groupes devraient être conduites.

Mots-clés : évaluation neuropsychologique, fonctions exécutives, dépendance aux drogues

Artigo recebido: 18/07/2011; Artigo revisado: 20/08/2011; Artigo aceito: 27/08/2011.

Hosana Alves Gonçalves, Faculdade de Psicologia, Pontifícia Universidade Católica do Rio Grande do Sul. Caroline de Oliveira Cardoso, Faculdade de Psicologia, Pontifícia Universidade Católica do Rio Grande do Sul. Renata Brasil Araújo, Hospital Psiquiátrico São Pedro.

Correspondências relacionadas a esse artigo devem ser enviadas a: Hosana Alves Gonçalves. Endereço: Av. Ipiranga, 6681, Prédio 11, sala 932, Partenon, Porto Alegre, Brasil, CEP 90619-900.

E-mail: hosana.goncalves@acad.pucrs.br

DOI: $10.5579 / \mathrm{rnl} .2011 .0070$ 


\section{Abstract}

The intersection of neuropsychology and psychopathology is still incipient, especially when it comes to the understanding of specific components of executive functions (EF) impaired by a neuropsychiatric disease as drug addiction. The present study aimed to characterize the cognitive profile of executive processing in a case of addiction to cocaine/crack. A flexible EF battery was used: Wisconsin Card Sorting Test - 48 cards, the Iowa Gambling Task, the Hayling Test and the three modalities of verbal fluency of the Bateria Montreal de Avaliação da Comunicação. The results suggest a preservation of the majority of EF components, such as verbal initiation and inhibition, attention, monitoring, planning and cognitive flexibility, but there seems to be a deficit in decisionmaking. The length of abstinence may have contributed to the spared performance of the patient in most tasks. Towards a better understanding of dissociations in executive processing in the neuropsychiatric disorder of drug addiction more multiple case studies and group studies need to be conducted.

Keywords: neuropsychological assessment; executive functions; substance dependence.

A neuropsicologia clínica tem crescido consideravelmente no Brasil sendo que a maioria dos estudos realizados nesta área foi feita com pacientes com quadros neurológicos (Bolognani, \& Fabrício, 2006; Camargo, Bolognani, \& Zuccolo, 2008; Fonseca, \& Parente, 2007). Embora se saiba que muitas alterações neuropsicológicas podem acompanhar um quadro psiquiátrico, a intersecção entre neuropsicologia e psicopatologia ainda se encontra muito incipiente tanto em nível nacional (Fonseca et al., 2010) quanto em nível internacional. Os dados são contraditórios e escassos principalmente no que tange ao entendimento mais específico de quais os processos cognitivos e componentes das funções executivas (FE) podem ser afetados por um determinado quadro neuropsiquiátrico.

Dentre os quadros psiquiátricos em que perfis neuropsicológicos deveriam estar sendo alvo de interesse desta área de interface entre psicopatologia e neuropsicologia destaca-se a dependência química. Tal realidade é observada em estudos internacionais, percebendo-se uma diversidade de pesquisadores interessados em estudar as funções cognitivas alteradas pelo uso de substâncias psicoativas (SPA), incluindo a memória, a capacidade atencional e as FE (Roberts, \& Garavan, 2010; Van der Plas et al., 2009; Chan, Shum, Toulopoulou, \& Chen, 2008; Pace-Schott et al., 2008; Verdejo-García, López-Torrecillas, Orozco, \& Péres-García, 2004). Na América Latina, embora seja evidente uma lacuna de estudos que se preocupem em analisar perfis executivos de dependentes de substâncias, alguns passos começam a ser dados para preenchê-la. Até onde se sabe, ainda são poucos os estudos publicados e estes avaliaram principalmente as FE em dependentes de álcool e maconha (Cunha, \& Novaes, 2004; Martínez, \& Manoiloff, 2010; Rigoni, Oliveira, Moraes, \& Zambom, 2007).

A cocaína é uma substância alcalóide utilizada mais comumente em sua forma aspirada (hidrocloridrato de cocaína) ou fumada (base livre de cocaína) que acarreta diversas complicações ao sistema nervoso central e a outros aspectos da saúde do usuário (Dias et al., 2011; Milroy, \& Parai, 2011). Sabe-se que a fórmula química da cocaína em pó e da pedra de crack é idêntica, o que difere é o método de preparação da substância (Treadwell, \& Robinson, 2006). A dependência da cocaína sob a forma do crack, mais especificamente, pode levar a graves e persistentes déficits neuropsicológicos em uma ampla gama de processos básicos (Di Sclafani, Tolou-Shams, Price, \& Fein, 2002). Um estudo realizado por Cunha, Nicastri, Gomes, Moino e Peluso (2004) com dependentes de cocaína/crack objetivou analisar as consequiências neurocognitivas decorrentes do uso desta substância avaliando habilidades atencionais, mnemônicas, executivas, lingüísticas e intelectuais, além de outros domínios como aprendizagem e funções visuo-espaciais, nota-se que a avaliação das FE não foi o foco principal deste estudo. Os resultados apontaram um baixo desempenho desta amostra nos testes que avaliavam atenção, FE, memória visual de longa duração e aspectos de aprendizagem verbal.

A maioria dos estudos internacionais que buscam avaliar as funções cognitivas comprometidas em usuários de crack é feita com usuários abstinentes comparados a controles com ou sem uso na vida de SPA. Estes estudos relatam pior desempenho em usuários de crack em tarefas que examinam atenção (componente de vigilância), tempo de reação, velocidade psicomotora e memória espacial e verbal (PaceSchott et al., 2005).

No que tange ao processamento atencional, um dos construtos mais investigados nesta população, Garavan e Hester (2007) reportam que usuários de cocaína sofrem alguns vieses atencionais e tem dificuldade em controlar o foco de sua atenção, o que pode contribuir, inclusive, para a própria procura pela droga. Em complementaridade, os achados neuroanatomofuncionais sobre as mudanças atencionais neste quadro vem evidenciando que há uma hipoativação de regiões pré-frontais e de núcleos da base, além de giro cingulado (Kübler, Murphy, \& Garavan, in press), áreas altamente relacionadas a FE. Segundo PaceSchott et al. (2005), durante a abstinência, o desempenho de usuários de crack em tarefas de vigilância deteriora-se, bem como a velocidade do processamento da informação e o desempenho nas tarefas de reconhecimento verbal imediato e tardio, porém, esse prejuízo não ocorre nas tarefas que avaliam memória de trabalho. Os dados sugerem que a abstinência pode tornar claro se os déficits cognitivos são ou não induzidos pelo uso crônico do crack. Entretanto, deve-se salientar que, mesmo após um longo tempo de abstinência, pode não haver melhoras significativas nas funções cognitivas desses indivíduos, não sendo descartada uma associação destas alterações com o uso dessa substância (Scheffer, Pasa, \& Almeida, 2009).

Além do consumo de drogas estar relacionado com a existência de alterações em distintos processos neuropsicológicos, incluindo mecanismos de atenção e memória, existem evidências empíricas, cada vez mais frequentes na literatura, que indicam que as alterações neurocognitivas associadas ao consumo de drogas afetam especialmente as FE (Bechara, 2005; Verdejo-García et al., 2004). De maneira geral, as FE são um conjunto de habilidades implicadas que gerenciam o comportamento de um indivíduo para que seja alcançada uma meta específica (Chan, Shum, Toulopoulou, \& Chen, 2008). Trata-se de um termo amplo e complexo que integra diversos componentes cognitivos, tais como, processos de resolução de problemas, 
controle inibitório, planejamento, flexibilidade cognitiva, tomada de decisão, entre outros (Damásio, 1995; Jurado \& Rosselli, 2007). Além disso, as FE necessitam do envolvimento de outros subcomponentes como atenção, inibição de processos e informações concorrentes e monitoramento (Kristensen, 2006). A síndrome disexecutiva, denominada por Baddeley (1986) para se referir a um conjunto de alterações de um ou mais componentes das FE, é uma das sequelas associadas a vários quadros psiquiátricos, inclusive à dependência química (Boller, \& Botez-Marquard, 2005; Caixeta et al, 2007).

Mais especificamente, quanto ao processamento de componentes das FE no caso de dependentes de substâncias estimulantes do Sistema Nervoso Central, segundo Bechara et al. (2001), pode haver prejuízo no processo de tomada de decisão, o que contribui para a manutenção das práticas abusivas. Estudos recentes vêm evidenciando alterações préfrontais em dependentes de cocaína/crack, relacionadas a déficits na tomada de decisões destes indivíduos (Cunha, Bechara, Andrade, \& Nicastri, 2011; Garavan, \& Hester, 2007). Outras pesquisas sugerem que usuários de cocaína/crack podem ter um controle inibitório deficiente e um comprometimento na avaliação de seus comportamentos de risco (Colzato, \& Hommel, 2009; Garavan, \& Hester, 2007), acarretando um déficit na sua capacidade de automonitoramento.

Aharonovich et al. (2008) alertam que os dependentes de cocaína/crack podem ter maior dificuldade de adesão e compromisso com o tratamento psicoterápico devido ao prejuízo em algumas habilidades cognitivas como a flexibilidade mental. A rigidez do pensamento pode dificultar, então, que o paciente selecione estratégias de enfrentamento adequadas às situações de risco, bem como pode prejudicar a manifestação de compromisso com a mudança.

As investigações internacionais parecem estar rumando para uma descrição cada vez mais específica do processamento executivo na abstinência de crack. Resta, ainda, a necessidade de maior investimento para além dos subprocessos tomada de decisão, inibição e flexibilidade mental, investigando a relação entre estes juntamente com outros componentes, tais como, planejamento, organização, executivo central da memória de trabalho, raciocínio, switching, clustering, abstração, entre outros. No Brasil, embora algumas pesquisas venham sendo conduzidas com dependentes de crack (Araujo, Pansard, Boeira, \& Rocha, 2010; Castro, Pedroso, \& Araujo, 2010) é evidente uma lacuna de estudos publicados com enfoque no desempenho destes indivíduos em tarefas que avaliam componentes das FE, ou seja, com uma abordagem neuropsicológica.

Os estudos publicados com dependentes químicos e com enfoque neuropsicológico são em sua maioria estudos de grupos. No entanto, sabe-se que pode haver diferenças significativas entre o desempenho cognitivo dos participantes de um grupo e que a homogeneidade dos casos nem sempre é real. Os estudos de caso são importantes ferramentas que auxiliam na compreensão de especificidades e permitem ao terapeuta testar as hipóteses relativas ao quadro em questão, confirmando ou reformulando-as, para então organizar um plano específico de reabilitação favorecendo assim o paciente (Kristensen, Almeida, \& Gomes (2001). Nesse contexto, o objetivo deste estudo é procurar, de modo preliminar, caracterizar o perfil cognitivo de processamento executivo em um dependente de crack no período pós-desintoxicação.

\section{Método}

\section{Delineamento}

Este artigo refere-se a um relato de caso de um paciente dependente de crack internado para desintoxicação.

\section{Caso Clínico}

O paciente avaliado - do sexo masculino, 30 anos de idade, solteiro, com o ensino médio completo, trabalha como vendedor - foi recrutado entre os pacientes internados na Unidade de Desintoxicação do Hospital Psiquiátrico São Pedro de Porto Alegre/RS/Brasil e tem diagnóstico de dependência de crack e maconha e de uso abusivo de álcool segundo os critérios do DSM-IV-TR (American Psychiatric Association- APA, 2002). O uso de SPA iniciou aos 14 anos de idade, quando experimentou bebida alcoólica pela primeira vez; com 19 anos experimentou maconha e, em seguida, a cocaína inalada. Iniciou o consumo de crack há 6 anos, quando tinha 24 anos de idade. No momento da avaliação, o paciente estava abstinente de maconha há aproximadamente 5 anos, caracterizando um quadro de dependência em remissão completa desta substância (APA, 2002), e estava há 22 dias sem consumir crack e álcool. Não apresenta nenhum outro diagnóstico de Eixo I além dos relativos ao uso de SPA (APA, 2002). Apresentou-se muito colaborativo tanto na entrevista diagnóstica quanto na sessão de avaliação, contribuindo e consentindo com a publicação deste trabalho.

\section{Instrumentos}

Para verificar os dados sociodemográficos e os hábitos e freqüência de uso de SPA utilizou-se uma entrevista semiestruturada. Para descartar possíveis comorbidades, realizou-se uma entrevista estruturada com base nos critérios do DSM-IV-TR (APA, 2002) por um estagiário de psicologia da unidade. Uma bateria de testes que avaliam as FE foi planejada, tomando-se o cuidado para utilizar instrumentos que avaliassem alguns subcomponentes deste construto maior e multidimensional:

1) Teste Wisconsin de Classificação de Cartas - 48 cartões (WCST-48 - Nelson, 1976). Este teste é uma medida de FE (planejamento, flexibilidade do pensamento, memória de trabalho, monitoramento, e inibição de perseverações), que requer a capacidade do examinando para desenvolver e manter uma estratégia apropriada de solução de problemas por meio de condições de estímulos mutáveis a fim de atingir uma meta futura. Ele avalia o raciocínio abstrato, a capacidade do examinando em gerar estratégias para resolução de problemas em situações de mudanças. $O$ instrumento é composto por quatro cartas-chave e 48 cartasresposta, que são apresentadas com figuras de diferentes formas (círculos, cruzes, triângulos ou estrelas), diferentes cores (azul, vermelho, amarelo ou verde) e diferentes quantidades (um, dois, três ou quatro). O examinando é solicitado a combinar as cartas-estímulo com as cartas-chave, sendo que, em cada combinação, recebe o feedback de certo ou errado do examinador. O objetivo é que o examinando possa utilizar o feedback recebido para auxiliar no desenvolvimento de novas estratégias na resolução dos problemas do instrumento (Heaton, Chelune, Talley, Kay, \& Curtiss, 1993). Neste teste considerou-se o número de erros e acertos, o número de ensaios administrados, de categorias 
completadas, de erros perseverativos e não perseverativos e o número de rupturas.

2) Iowa Gambling Task (IGT) - (Bechara, Damásio, Damásio, \& Anderson, 1994; Schneider, \& Parente, 2006). Foi aplicada uma versão computadorizada da IGT, a partir da versão atualmente utilizada pelo autor do instrumento e que foi adaptada para o Português Brasileiro por Schneider e Parente (2006). A tarefa envolve um jogo de cartas e avalia o processo de tomada de decisão do indivíduo em termo de busca ou aversão ao risco. Este instrumento avalia o comportamento do indivíduo em um jogo de cartas composto por quatro baralhos. Em dois dos baralhos (A e B), muito dinheiro é ganho a curto prazo, mas muito dinheiro é perdido a longo prazo, sendo considerados baralhos de risco; nos outros dois baralhos (C e D), o avaliando ganha pouco dinheiro a curto prazo, mas perde ainda menos, sendo estes mais vantajosos a longo prazo. A conduta do paciente foi analisada através do cálculo global e do cálculo por blocos. A primeira análise é derivada a partir do cálculo envolvendo a subtração do número de cartas retiradas dos baralhos vantajosos, daqueles retiradas dos baralhos desvantajosos $[(\mathrm{C}+\mathrm{D})-(\mathrm{A}+\mathrm{B})]$ e a segunda, denominada cálculo por blocos, refere-se ao escore obtido em cada um dos cincos blocos de 20 cartas, ao longo de 100 jogadas. O cálculo utilizado para obtenção do resultado por blocos é o mesmo realizado no cálculo global, realizado a cada bloco de 20 jogadas. As médias de cada segmento são consideradas para observar a curva de aprendizagem ao longo da tarefa. Esse instrumento contempla uma situação de tomada de decisão sob incerteza, que envolve escolhas monetárias em curto e longo prazo, permitindo classificar o comportamento de decisão do indivíduo em termos de uma habilidade de decisão adaptativa ou prejudicada (Bechara, Damásio, Damásio, \& Anderson, 1994; Schneider, \& Parente, 2006).

3) Fluência Verbal (livre, com critério ortográfico e semântico) (Bateria Montreal de Avaliação da Comunicação Bateria MAC (Fonseca, Parente, Côté, Ska, \& Joanette, 2008). Os componentes cognitivos examinados nesta tarefa são planejamento, iniciação e inibição verbais, memória lexical, memória de trabalho e flexibilidade cognitiva. $\mathrm{Na}$ modalidade livre, o participante é solicitado a evocar todas as palavras que lembrar, desde que não seja nomes próprios nem números, por dois minutos e meio. Na fluência ortográfica, deve evocar palavras que começam com a letra 'p', em dois minutos e, na semântica, palavras que sejam roupas/vestimentas, em dois minutos. Contabilizou-se a quantidade de palavras corretas em cada bloco de 30 segundos para cada modalidade da tarefa.

4) Hayling Test (Fonseca et al., 2010). O Hayling Test (Burguess, \& Shallice, 1997) adaptado para o português brasileiro por Fonseca et al. (2010) é um teste de completar sentenças nas quais falta a última palavra. O teste é composto de duas partes: na parte $\mathrm{A}$, o indivíduo deve produzir a palavra que completa de maneira coerente a frase / sentença. Na parte B, o participante deve produzir uma palavra que não apresente relação com a frase. Na parte A, o contexto sintático e semântico do estímulo (sentença) conduz a ativação de uma palavra coerente com o campo semântico da oração. Na parte $\mathrm{B}$, o indivíduo deve inibir a resposta dominante (lógica, coerente) e buscar uma palavra não relacionada com o contexto sintático e semântico que a oração impõe. Com este teste é possível avaliar componentes atencionais e outras funções cognitivas como memória semântica, iniciação e inibição verbal, planejamento e velocidade de processamento. Nesta tarefa considerou-se o tempo em segundos, o número de acertos e de erros tanto para sua parte A quanto para a parte $\mathrm{B}$, além destas variáveis foram considerados o número de erros com a categorização das frases e o escore de tempo parte B - parte A.

\section{Procedimentos}

Este trabalho foi planejado de acordo com a Resolução 196/96 do Conselho Nacional de Saúde que regula as pesquisas envolvendo seres humanos. O participante ingressou nessa pesquisa mediante explícita autorização por sua assinatura a um Termo de Consentimento Livre e Esclarecido, após o projeto ter sido aceito pelo Comitê de Ética em Pesquisa da instituição (CEP n ${ }^{\circ}$ 11017).

A bateria de testes neuropsicológicos foi aplicada após duas semanas de internação. Os testes foram aplicados, individualmente, em uma única sessão de aproximadamente 1 hora e 20 minutos, em ambiente apropriado para tal. As tarefas verbais foram registradas em gravador de voz para posterior transcrição e análise, sendo todo e qualquer arquivo de áudio destruído após transcrição. Em algumas tarefas, foi avaliada a velocidade de processamento do participante, sendo necessário o uso de um cronômetro digital.

\section{Análise de dados}

Foram efetuadas análises a partir do escore Z (escore do caso - média do grupo normativo/desvio-padrão (DP) do grupo normativo), utilizando o ponto de corte sugerido pela literatura de $\mathrm{Z} \leq-1,5$ (Kavé, Heled, Vakil, \& Agranov, 2010). Os resultados obtidos foram analisados à luz do referencial teórico pesquisado.

\section{Resultados}

Os principais resultados do desempenho executivo do paciente por instrumento da bateria de avaliação neuropsicológica são apresentados em duas tabelas e uma figura.

A Tabela 1 apresenta os resultados obtidos pelo participante no WCST-48 e no Hayling Test.

Tabela 1

Desempenho do paciente no Teste Wisconsin 48 cartões e no Hayling Test

\begin{tabular}{|c|c|c|c|}
\hline Instrumento & Variáveis & Pontuação & $\begin{array}{l}\text { Escore } \\
\mathrm{Z}\end{array}$ \\
\hline \multirow{3}{*}{$\begin{array}{l}\text { Teste Wisconsin } \\
-48 \text { cartas }\end{array}$} & $\begin{array}{l}\text { Categorias } \\
\text { completadas }\end{array}$ & 3 & $-0,91$ \\
\hline & $\begin{array}{c}\text { Erros não } \\
\text { perseverativos }\end{array}$ & 7 & $-0,80$ \\
\hline & $\begin{array}{c}\text { Erros } \\
\text { perseverativos }\end{array}$ & 13 & $-0,36$ \\
\hline \multirow{5}{*}{ Hayling Test } & Parte A erros/15 & 0 & 0,15 \\
\hline & Parte A tempo(s) & 7’09 & 1,51 \\
\hline & Parte B erros/15 & 2 & 0,67 \\
\hline & Parte B tempo(s) & 33’19 & 0,21 \\
\hline & Parte B erros/45 & 4 & 0,86 \\
\hline
\end{tabular}


A seguir, a Tabela 2 apresenta os resultados nas diferentes modalidades da tarefa de fluência verbal.

Tabela 2

Desempenho do paciente nas tarefas de Fluência Verbal

\begin{tabular}{clcc}
\hline Critério da Tarefa & \multicolumn{1}{c}{ Variáveis } & Pontuação & $\begin{array}{c}\text { Escore } \\
\mathrm{Z}\end{array}$ \\
\hline \multirow{4}{*}{ Fluência Verbal Livre } & Bloco 0-30 s & 12 & $-0,78$ \\
& Bloco 30-60 s & 10 & $-0,58$ \\
& Bloco 60-90 s & 13 & 0,28 \\
& Bloco 90-120 s & 9 & $-0,22$ \\
& Bloco 120-150 s & 12 & 0,40 \\
\hline \multirow{2}{*}{ Fluência Verbal com } & Bloco 0-30 s & 8 & $-1,21$ \\
Critério Ortográfico & Bloco 30-60 s & 2 & $-2,10$ \\
(P) & Bloco 60-90 s & 4 & $-0,35$ \\
& Bloco 90-120 s & 5 & 0,31 \\
\hline \multirow{2}{*}{ Fluência Verbal com } & Bloco 0-30 s & 10 & $-1,45$ \\
critério semântico & Bloco 30-60 s & 5 & $-0,88$ \\
(Roupas/vestimentas) & Bloco 60-90 s & 4 & $-0,27$ \\
& Bloco 90-120 s & 6 & 1,15 \\
\hline
\end{tabular}

Já na análise do cálculo global do IGT, derivado da soma das escolhas dos baralhos vantajosos menos o número de cartas retiradas dos baralhos desvantajosos, o paciente obteve um escore -23 . Baseando-se nas normas internacionais de classificação de desempenho, o paciente apresentou um desempenho prejudicado no processo de tomada de decisão. A Figura 1, em complementaridade, apresenta os resultados do cálculo por blocos do participante durante a tarefa do IGT.

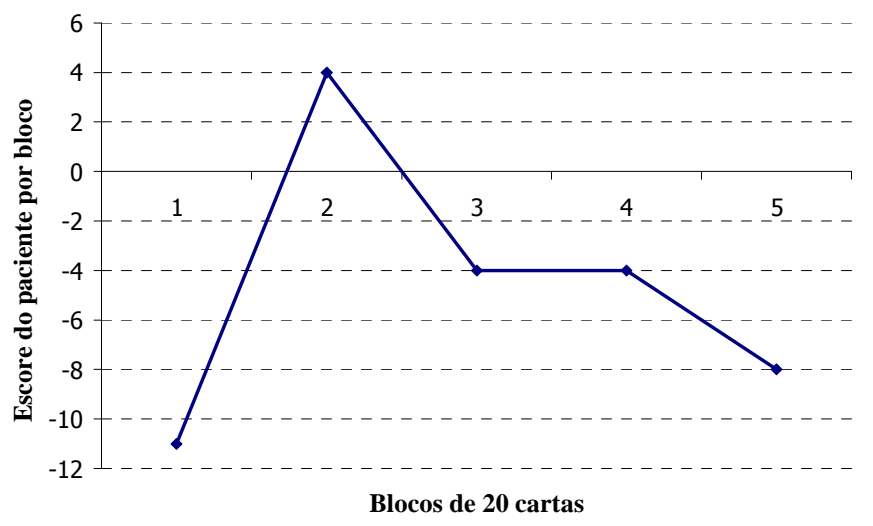

Figura 1. Desempenho do paciente em cada bloco do IGT

Diante dessa figura, evidencia-se que não houve uma curva ascendente de aprendizagem ao longo da tarefa. Apesar de demonstrar uma melhora do primeiro para o segundo bloco ao longo das jogadas, seu desempenho sofreu uma considerável redução posteriormente, apresentando uma tendência a escolher mais cartas dos baralhos de risco. Concomitantemente à análise quantitativa, após a aplicação do teste, questionou-se ao paciente se ele havia utilizado alguma estratégia específica e se havia identificado os baralhos de risco. Sua resposta foi positiva e o mesmo assinalou, de forma correta, que os baralhos A e B eram desvantajosos, contudo, verbalizou que preferiu se manter usando as cartas em questão por serem as que mais ofereciam chances de ganhar uma maior quantia de dinheiro, embora

Revista Neuropsicologia Latinoamericana (2011), 3(2), 7-13 tivesse grandes probabilidades de perder uma quantidade maior de dinheiro

\section{Discussão}

A proposta desse estudo foi caracterizar o perfil executivo de um paciente dependente de crack no período pós-desintoxicação. $\mathrm{O}$ seu desempenho nas diferentes modalidades de fluência verbal não se mostrou deficitário com relação ao do seu grupo normativo, o que pode indicar uma preservação na capacidade de iniciação e inibição verbal, bem como de memória semântica. Tal achado está de acordo com os resultados de Cunha et al. (2004), cujos participantes obtiveram um padrão de desempenho semelhante ao do grupo controle em tarefas de fluência verbal com critério semântico (animais, comida e roupas). Porém, no segundo bloco de tempo da Fluência Verbal com critério ortográfico (30-60 segundos), percebe-se que o desempenho do paciente foi abaixo do esperado para a sua faixa etária e seu nível de escolaridade, ou seja, ele evocou menos palavras do que o esperado em relação ao grupo normativo. Esse dado corrobora os achados de Cunha et al. (2004), que encontraram diferenças estatisticamente significativas nos testes de fluência verbal fonológica em usuários de cocaína/crack, e os de Fernández-Serrano et al. (2010) que evidenciaram uma diferença significativa entre desempenho de grupos-controle e de dependentes de substâncias na mesma modalidade de uma tarefa de fluência verbal. Contudo, não se pode afirmar que o paciente em questão tenha déficits significativos de evocação de palavras a partir de um critério fonológico, uma vez que seu desempenho se mostrou rebaixado apenas em um dos blocos de tempo da tarefa.

Quando se leva em consideração os resultados do paciente no Hayling Test, percebe-se uma preservação das habilidades de monitoramento, atenção concentrada, iniciativa verbal, planejamento, velocidade de processamento e inibição verbal demandadas pela tarefa. Estes dados podem ser observados tanto nas respostas dadas como no tempo de execução da tarefa. Um estudo de Scheffer, Pasa e Almeida (2009) confirma nossos achados no que diz respeito ao componente atencional avaliado pelo Hayling Test. Neste estudo, realizado com 17 dependentes de cocaína/crack e 15 dependentes de álcool e cocaína/crack, não foi encontrada diferença significativa nos níveis de atenção concentrada quando se comparou os dois grupos ao grupo-controle.

No que diz respeito à inibição, Garavan e Hester (2007) verificaram que estudos de neuroimagem mostram um controle inibitório prejudicado em tarefas de inibição motora e do tipo Go/noGo em usuários de SPA estimulantes ainda intoxicados, porém, assim como em nosso estudo, Van der Plas, Crone, Van der Wildenberg, Tranel e Bechara (2009) não encontrou prejuízos em tarefas de inibição de resposta em dependentes de psicoestimulantes. O resultado apresentado anteriormente por Garavan e Hester (2007) pode não ter se repetido pelo fato do participante deste estudo de caso não estar sob efeito de SPA, já estando desintoxicado, assim como os participantes do estudo de Van der Plas et al. (2009).

A falha no monitoramento do comportamento também é um fenômeno que pode ser característica de abuso de drogas em geral (Fernandez-Serrano et al., 2010), porém o paciente avaliado, neste estudo, não demonstrou prejuízo neste subcomponente complexo das FE. A preservação de algumas funções cognitivas no paciente em questão pode confirmar a hipótese de alguns autores que defendem que, 
com a abstinência, é possível que haja melhora em algumas habilidades cognitivas prejudicadas pelo uso da substância (Di Sclafani et al., 2002; Selby, \& Azrin, 1998). Os resultados apresentados até então sugerem que o paciente teve resultado satisfatório, apontando para uma preservação de alguns componentes das FE.

Além disso, observa-se que, quando comparado ao grupo normativo, o paciente teve um bom desempenho no WCST - 48 cartões, o que sugere uma preservação em alguns subcomponentes de FE, como planejamento, capacidade de escolha por uma estratégia e manutenção na mesma enquanto ela é efetiva, bem como mudança de estratégia quando ela não é mais útil (flexibilidade cognitiva). Os achados de Cunha et al (2004) e de Cunha, et al. (2011) corroboram os do presente estudo de caso, uma vez que, nestes dois estudos, não houve diferenças significativas no desempenho de dependentes de cocaína/crack no WCST quando comparados ao grupo de pessoas saudáveis. Van der Plas et al. (2009) também não encontraram prejuízos no desempenho de dependentes químicos de álcool, cocaína e metanfetamina nesta tarefa. Aharonovich et al. (2006) discutem a sensibilidade do WCST para detectar a especificidade e os processos cognitivos subjacentes aos quadros onde os danos não são tão graves como no uso de cocaína, uma vez que os prejuízos executivos ficam evidenciados em outras tarefas de FE como o IGT, mas não no WCST. Estudos com grupos são necessários para analisar estes achados, uma vez que este estudo de caso não tem a pretensão de generalizar os resultados, embora respaldados pela literatura já existente.

Em relação ao IGT, baseando-se nas normas internacionais de classificação de desempenho, o paciente apresentou um desempenho prejudicado no processo de tomada de decisão. No cálculo por blocos, evidencia-se que não houve uma curva ascendente de aprendizagem ao longo da tarefa e que o participante apresentou uma tendência a escolher mais cartas dos baralhos desvantajosos. Estes resultados são semelhantes aos achados de Grant, Contoreggi e London (2000), que também observaram uma tendência de dependentes químicos em utilizarem mais baralhos de risco em detrimento dos vantajosos. Junto a isso, Bechara e Damásio (2002) evidenciaram que os dependentes de drogas, de forma geral, apresentam alterações na geração de representações emocionais associadas às distintas alternativas de resposta do IGT e outras tarefas de tomada de decisão. Já a verbalização do paciente sobre a estratégia utilizada durante o jogo vai ao encontro do que Bechara, Damasio, Tranel e Damasio (2005) constataram ao examinar alguns pacientes que sofreram lesão pré-frontal ventromedial. Segundo os autores, muitos pacientes são capazes de evocar o conhecimento factual da tarefa, porém, continuam perseverando na escolha por baralhos desvantajosos. Essa contradição também pode ser observada nas dificuldades dos dependentes químicos no controle de suas respostas emocionais, nas suas altas taxas de recaída, na sua baixa adesão ao tratamento, no seu envolvimento em situações de risco e no seu desejo por recompensa à curto prazo, o que já foi descrito por Kolling et al. (2007) e por Cunha, Nicastri, Gomes, Moino e Paluso (2011). Cientes desta tendência, terapeutas das mais diversas linhas teóricas e profissionais que trabalham com reabilitação neuropsicológica podem integrar a reabilitação e treinamento de habilidades de tomada de decisão no tratamento da dependência química.
A maioria dos componentes executivos avaliados parecem estar preservados no paciente estudado, o que vai ao encontro de alguns achados na literatura nacional e internacional, mas que também se contrapõe a outros estudos. O tempo de abstinência do paciente pode ter contribuído para esse bom desempenho, corroborando com pesquisas que confirmam o restabelecimento de funções cognitivas após determinado tempo de abstinência. Deve-se destacar que o paciente avaliado também era dependente de maconha (porém, abstinente há 5 anos) e fazia uso abusivo de álcool, o que pode ter interferido nos resultados, no entanto, a prática clínica mostra que o uso de poli-substâncias é algo que não se consegue evitar quando se pesquisa dependentes de cocaína/crack.

O presente artigo, sendo um estudo de caso, não tem a pretensão de generalizar seus resultados, por isso sugere-se que sejam realizadas novas pesquisas com amostras maiores para compreender melhor como se dá esta reorganização cognitiva de dependentes de crack após o período de desintoxicação.

\section{Referências}

Aharonovich, E., Hasin, D.S., Brooks, A.C., Liu, X., Bisaga, A., \& Nunes, E.V. (2006). Cognitive deficits predict low treatment retention in cocaine dependent patients. Drug and Alcohol Dependence, 81, 313322 .

Aharonovich, E., Amrhein, P. C., Bisaga, A., Nunes, E. V., \& Hasin, D. S. (2008). Cognition, Commitment Language, and Behavioral Change Among Cocaine-Dependent Patients. Psychology of Addictive Behaviors, 22 (4), 557-562.

American Psychiatric Association. (2002). DSM - IV - TR: Manual Diagnóstico e Estatístico de Transtornos Mentais. Dornelles, C. (Trad.) $4^{\text {a }}$ ed. Porto Alegre: Artmed.

Araujo, R. B., Pansard, M., Boeira, B. U., \& Rocha, N. S. (2010). As Estratégias de coping para o manejo da fissura de dependentes de crack. Revista HCPA, 30, 36-42.

Baddeley, A. (1986). Working memory. Oxford: Oxford University Press

Bechara, A., Damásio, A., Damásio, H., \& Anderson, S. (1994). Insensitivity to future consequences following damage to human prefrontal cortex. Cognition, 50, 7-15.

Bechara, A., \& Damásio, H. (2002). Decision-making and addiction (part I): impaired activation of somatic states in substance dependent individuals when pondering decisions with negative future consequences. Neuropsychologia, 40, 1675-1689.

Bechara, A., Damásio, H., Tranel, D., \& Damásio, A. R. (2005). The Iowa Gambling Task and the somatic marker hypothesis: some questions and answers. Trends in Cognitive Sciences, 9 (4), 159-162.

Bechara, A., Dolan, S., Denburg, N., Hindes, A., Anderson, S. W., \& Nathan, P. E. (2001). Decision-making deficits, linked to a dysfunctional ventromedial prefrontal cortex, revealed in alcohol and stimulant abusers. Neuropsychologia, 39, 376-389.

Boller, F., \& Botez-Marquard, T. (2005). Neuropsychologie Clinique et neurologie du comportement. Canadá: PUM.

Bolognani, S. A. P., \& Fabrício, A. M. (2006). Reabilitação neuropsicológica em lesão cerebral adquirida: o desafio de trabalhar com a diversidade. Em J. Abrisqueta-Gomez, \& F. H. Santos (Eds.), Reabilitação Neuropsicológica da Teoria à Prática (pp. 97-110). Porto Alegre: Artes Médicas.

Burgess, P. W., \& Shalice, T. (1997). The Hayling and Brixton Tests. Thurston, Suffolk:

Thames Valley Test Company.

Caixeta, M., Costa, F. C. O., Caixeta, L., Nóbrega, M., \& Hanna, M. (2007). Neuropsicologia dos Transtornos Mentais. São Paulo: Artes Médicas.

Camargo, C. H. P., Bolognani, S. A. P., \& Zuccolo, P. F. (2008). O exame neuropsicológico e os diferentes contextos de aplicação. Em Fuentes, D., Consenza, R. M., Malloy-Diniz, L. F., \& Camargo, C. H. P. (Eds.). Neuropsicologia: teoria e prática, 103-118. Porto Alegre: Artmed.

Castro, M. G. T., Pedroso, R. S., \& Araujo, R. B. (2010). Dependentes de crack com sintomas de transtorno de déficit de atenção/hiperatividade consomem mais substâncias psicoativas. Revista HCPA, 30, 118-124. 
Chan, R., Shum, D., Toulopoulou, T. \& Chen, E. (2008). Assessment of executive functions: Review or instruments and identification of critical issues. Archives of Clinical Neuropychology, 23, 201-216.

Colzato, L. S., \& Hommel, B. (2009). Recreational Use of Cocaine Eliminates Inhibition of Return. Neuropsychology, 23 (1), 125-129.

Cunha, P. J., Bechara, A., Andrade, A. G., \& Nicastri, S. (2011). DecisionMaking Deficits Linked to Real-life Social Dysfunction in Crack Cocaine-Dependent Individuals. American Journal Addiction, 20 (1), 78-86.

Cunha, P.J., Nicastri, S., Gomes, L. P., Moino, R. M., \& Peluso, M. A. (2004). Neuropsychological impairments in crack cocaine-dependent inpatients: preliminary findings. Revista Brasileira de Psiquiatria, 26 (2), 103-106.

Cunha, P.J., \& Novaes, M.A. (2004). Avaliação neurocognitiva no abuso e dependência do álcool: implicações para o tratamento. Revista Brasileira de Psiquiatria, 26 (1), 23-27.

Di Sclafani, V., Tolou-Shams, M., Price, L. J., \& Fein, G. (2002). Neuropsychological performance of individuals dependent on crackcocaine, or crack-cocaine and alcohol, at 6 weeks and 6 months of abstinence. Drug and Alcohol Dependence, 66, 161-171.

Fernández-Serrano, M. J., Pérez-García, M., Río-Valle, J. S., \& VerdejoGarcía, A. (2010). Neuropsychological consequences of alcohol and drug abuse on different components of executive functions. Journal of Psychopharmacology, 24 (9), 1317- 1332.

Fonseca, R. P., Oliveira, C., Gindri, G., Zimmermann, N., \& Reppold, C. (2010). Teste Hayling: um instrumento de avaliação de componentes das funções executivas. Em Hutz, C. (Org.). Avaliação Psicológica e Neuropsicológica de crianças e adolescentes. São Paulo: Casa do Psicólogo.

Fonseca, P. F., \& Parente, M. A. M. P. (2007). Metanálise de estudos do processamento comunicativo em indivíduos com lesão vascular direita. Estudos de Psicologia, 24(4), 529-538.

Fonseca, R. P., Parente, M. A. M. P., Côté, H., Ska, B., \& Joanette, Y. (2008). Bateria Montreal de Avaliação da Comunicação - Bateria MAC. Pró-Fono. São Paulo

Garavan, H., \& Hester, R. (2007). The Role of Cognitive Control in Cocaine Dependence. Neuropsychology, 17, 337-345.

Gonzales, R., Vassileva, J. \& Scott, C. (2009). Neuropsychological Consequences of drug abuse. Em Grant, I., \& Adams, K. M. Neuropsychological Assessment of Neuropsychiatric and Neuromedical Disorders. 3rd ed. New York: Oxford University Press.

Grant, S., Contoreggi, C., \& London, E. D. (2000). Drug abusers show impaired performance in a laboratory test of decision making. Neuropsychology, 38 (8), 1180-1187.

Heaton, R. K., Chelune, G. J., Talley, J. L., Kay, G. G., Curtiss, G. (1993). Wisconsin Card Sorting Test (WCST) manual, revised and expanded. Odessa, FL: Psychological Assessment Resources.

Jurado, M. B, \& Rosselli, M. (2007). The elusive nature of executive functions: A review of our current understanding, Neuropsychology Review, 17, 213-233.

Kavé, G., Heled, E., Vakil, E., \& Agranov, E. (2010). Which verbal fluency measure is most useful in demonstrating executive deficits after traumatic brain injury? Journal of Clinical and Experimental Neuropsychology, 1 (1), 1-8.

Kolling, N. M., Silva, C. R., Carvalho, J. C. N., Cunha, S. M., \& Kristensen, C. H. (2007). Avaliação neuropsicológica em alcoolistas e dependentes de cocaína. Avaliação Psicológica, 6 (2),127-137.

Kristensen, C.H., Almeida, R.M.M., \& Gomes, W.B. (2001). Desenvolvimento Histórico e Fundamentos Metodológicos da Neuropsicologia Cognitiva. Psicologia: Reflexão e Crítica,14(2), 259-274.

Kristensen, C. H. (2006). Funções executivas e envelhecimento. Em Parente, M. A. M. P. \& cols. (Eds.). Cognição e Envelhecimento. Porto Alegre: Artmed. $312 \mathrm{p}$

Kübler, A., Murphy, K., \& Garavan, H. (in press). Cocaine dependence and attention switching within and between verbal and visuospatial working memory. Euuropean Journal of Neuroscience. Retirado de http://www.drugsandalcohol.ie/6789/1/Garavan_3100_European_Jo urnal of Neuroscience.pdf.

Lezak, M. D. (1995). Neuropsychological Assessment. New York: Oxford University Press.

Martínez, M.V., \& Manoiloff, L.M.V. (2010). Evaluación Neuropsicológica de la Función Ejecutiva en Adolescentes con Diferentes Patrones de Consumo de Alcohol. Revista Argentina de Ciencias del Comportamiento, 2 (1), 14-23.
Milroy, C. M., \& Parai, J. L. (2011). The histopathology of drugs of abuse. Histopathology. Retirado http://www.ncbi.nlm.nih.gov/pubmed/21261690.

Nelson, H. E. (1976). A modified card sorting test sensitive to frontal lobe defects. Cortex, 12, 313-324.

Pace-Schott, E. F., Stickgold, R., Muzur, A., Wigren, P. E., Ward, A. S., Hart, C. L., Walker, M., Chris Edgar, C., \& Hobson, J. A. (2005). Cognitive Performance by Humans During a Smoked Cocaine Binge-Abstinence Cycle. American Journal of Drug and Alcohol Abuse, 31, 571-591.

Rigoni, M.S., Oliveira, M.S., Moraes, J.F.D., \& Zambom, L.F. (2007). O consumo de maconha na adolescência e as conseqüências nas funções cognitivas. Psicologia em Estudo, 12 (2), 267-275.

Rodrigues, V. S., Caminha, R. M., \& Horta, R. L. (2006). Déficits cognitivos em pacientes usuários de crack. Revista Brasileira de Terapias Cognitivas, 2(1).

Scheffer, M., Pasa, G. G., \& Almeida, R. M. M. (2009). Atenção, ansiedade e raiva em dependentes químicos. Psico PUCRS, 40 (2), 235-244.

Schneider, D., \& Parente, M. A. (2006). O desempenho de adultos jovens e idosos na Iowa Gambling Task (IGT): um estudo sobre a tomada de decisão. Psicologia: Reflexão e Crítica, 19 (3), p. 442-450.

Selby, M. J., \& Azrin, R. L. (1998). Neuropsychological functioning in drug abusers. Drug Alcohol Dependence, 50, 39-45.

Turner, T. H., LaRowe, S., Horner, M. D., Herron, J., \& Malcolm, R. (2009). Measures of cognitive functioning as predictors of treatment outcome for cocaine dependence. Journal of Substance Abuse Treatment, 37, 328-334.

Van der Plas, E. A. A., Crone, E. A., Van den Wildenberg, W. P. M., Tranel, D., \& Bechara, A. (2009). Executive control deficits in substancedependent individuals: A comparison of alcohol, cocaine, and methamphetamine and of men and women. Journal of Clinical and Experimental Neuropsychology, 31 (6), 706-719.

Verdejo-García, A., López-Torrecillas, F., Orozco, C., \& Pérez-García, M. (2004). Clinical implications and methodological challenges in the study of the neuropsychological correlates of cannabis, stimulant and opioid abuse. Neuropsychology Review, 14, 1-41. 